\title{
DOMINATION OF THE SUPREMUM OF A BOUNDED HARMONIC FUNCTION BY ITS SUPREMUM OVER A COUNTABLE SUBSET
}

\author{
by F. F. BONSALL
}

(Received 11th April 1986)

\section{Introduction}

For what sequences $\left\{a_{n}\right\}$ of points of the open unit disc $D$ does there exist a constant $\kappa$ such that

$$
\sup _{z \in D}|f(z)| \leqq \kappa \sup _{n \in \mathbb{N}}\left|f\left(a_{n}\right)\right|
$$

for all bounded harmonic functions $f$ on $D$ ?

This question is of interest because these are the sequences such that every integrable function $f$ on the unit circle $\partial D$ is of the form

$$
f=\sum_{n=1}^{\infty} \lambda_{n} p_{a_{n}}
$$

with $\sum_{n=1}^{\infty}\left|\lambda_{n}\right|<\infty$ (see [1]). Here

$$
p_{a}(\zeta)=\left(1-|a|^{2}\right)|1-\bar{a} \zeta|^{-2} \quad(\zeta \in \partial D, a \in D)
$$

that is $p_{a}\left(e^{i \theta}\right)$ is the Poisson kernel $P_{a}(\theta)$.

Brown, Shields and Zeller [2] have proved the closely related result that

$$
\sup _{z \in D}|f(z)|=\sup _{n \in \mathbb{N}}\left|f\left(a_{n}\right)\right|
$$

for all $f \in H^{\infty}$ (the space of bounded analytic functions on $D$ ) if and only if $\left\{a_{n}\right\}$ is nontangentially dense for $\partial D$, that is if and only if almost every point of $\partial D$ is the nontangential limit of some subsequence of $\left\{a_{n}\right\}$. Our main result, Theorem 2 , is a list of equivalent conditions on the sequence $\left\{a_{n}\right\}$ which includes conditions (1) and (3).

In Theorem 3, we establish an elementary property of the harmonic measure $\chi_{F}(z)$ of a Lebesgue measurable subset $F$ of $\mathbb{R}$; namely, $\chi_{F}(z)$ is arbitrarily small outside the union of certain triangular domains associated with the points of $F$. This shows that if the inequality (1) holds for all positive bounded harmonic functions, then $\left\{a_{n}\right\}$ is nontangentially dense. 
Theorem 2 describes the sequences $\left\{a_{n}\right\}$ for which the bounded linear mapping $T$ of $l^{1}$ into $L^{1}$ given by $T\left\{\lambda_{n}\right\}=\sum_{n=1}^{\infty} \lambda_{n} p_{a_{n}}$ is surjective. It is an immediate consequence that $T$ is never bijective. When is it injective? This question remains unanswered, but Theorem 6 shows that $T$ has zero kernel and closed range if and only if $\left\{a_{n}\right\}$ is an interpolating sequence for $H^{\infty}$.

I am indebted to W. K. Hayman for asking a question that provoked this work and also for an observation showing that there are no sequences $\left\{a_{n}\right\}$ for which the infimum in Theorem 2(ii) is always attained.

\section{Results}

In the following elementary lemma, $G$ denotes a simply connected domain in the complex plane, $H^{\infty}(G)$ the space of bounded analytic functions on $G$, and $B H(G)$ the space of bounded complex valued harmonic functions on $G$.

Lemma 1. Let $A$ be a subset of $G$, and let there exist a constant $\kappa$ such that

$$
\sup _{z \in \mathbf{G}}|f(z)| \leqq \kappa \sup _{z \in A}|f(z)|
$$

for all invertible elements $f$ of $H^{\infty}(G)$. Then

$$
\sup _{z \in G}|f(z)|=\sup _{z \in A}|f(z)|
$$

for all $f \in B H(G)$.

Proof. Let $u$ be a non-negative real valued element of $B H(G)$. Since $G$ is simply connected, there exists a function $g$ analytic on $G$ with $\operatorname{Re} g=u$. Let

$$
f(z)=\exp g(z) \quad(z \in G)
$$

Since $|f(z)|=\exp u(z)$, we have $f \in H^{\infty}(G)$, and plainly $1 / f$ is also in $H^{\infty}(G)$. Therefore inequality (4) holds, that is

$$
\underset{z \in G}{\sup } \exp u(z) \leqq \kappa \sup _{z \in A} \exp u(z)
$$

Therefore

$$
\sup _{z \in G} u(z) \leqq \log \kappa+\sup _{z \in A} u(z)
$$

This inequality also holds with $u$ replaced by $\alpha u$ with positive $\alpha$, and so

$$
\sup _{z \in G} u(z) \leqq \frac{1}{\alpha} \log \kappa+\sup _{z \in A} u(z)
$$


Therefore, $u$ satisfies (5). Next, if $h$ is any real valued bounded harmonic function on $G$, then $M \pm h$ is non-negative for suitable positive $M$, and so $h$ satisfies (5). Finally, given any complex valued $f \in B H(G)$, and $\theta \in \mathbb{R}$, let $h_{\theta}(z)=\operatorname{Re}\left(e^{i \theta} f(z)\right)$. Then $h_{\theta}$ satisfies (5). We choose $z_{0}$ in $G$ with $\left|f\left(z_{0}\right)\right|$ close to $\sup _{z \in G}|f(z)|$, and then choose $\theta$ so that $h_{\theta}\left(z_{0}\right)=\left|f\left(z_{0}\right)\right|$ to complete the proof.

In the following theorem, we write $L^{p}$ for $L^{p}(\partial D, d \theta / 2 \pi)$, and $H^{\infty}$ for $H^{\infty}(D)$.

Theorem 2. Given a sequence $\left\{a_{n}\right\}$ of points of $D$, the following conditions are equivalent to each other.

(i) Every $f \in L^{1}$ is of the form (2) with $\sum_{n=1}^{\infty}\left|\lambda_{n}\right|<\infty$.

(ii) Condition (i) holds and also

$$
\|f\|_{1}=\inf \sum_{n=1}^{\infty}\left|\lambda_{n}\right|
$$

with the infimum taken over all sequences $\left\{\lambda_{n}\right\}$ satisfying (2).

(iii) There exists a constant $\kappa$ such that the inequality (1) holds for all $f \in B H(D)$.

(iv) The equality (3) holds for all $f \in B H(D)$.

(v) The equality (3) holds for all $f \in H^{\infty}$.

(vi) Almost every point of $\partial D$ is the non-tangential limit of some subsequence of $\left\{a_{n}\right\}$.

Proof. The order of proof is (i) $\rightarrow$ (iii) $\rightarrow$ (iv) $\rightarrow$ (v) $\rightarrow$ (vi) $\rightarrow$ (ii) $\rightarrow$ (i).

(i) $\rightarrow$ (iii). Suppose that (i) holds, and, given $\lambda=\left\{\lambda_{n}\right\} \in l^{1}$, let

$$
T \lambda=\sum_{n=1}^{\infty} \lambda_{n} p_{a_{n}} .
$$

Since $\left\|p_{a}\right\|_{1}=1, T$ is a bounded linear mapping of $l^{1}$ onto $L^{1}$. It is therefore an open mapping, and there exists $\kappa>0$ such that the image of the ball in $l^{1}$ with centre 0 and radius $\kappa$ contains the unit ball in $L^{1}$. Thus (2) holds for all $f \in L^{1}$, and

$$
\inf \left\{\|\lambda\|_{1}:(2) \text { holds }\right\} \leqq \kappa\|f\|_{1} \text {. }
$$

Now let $g \in L^{\infty}$ with $g(z)$ its harmonic extension to $D$, and let $\varepsilon>0$. Since $\|g\|_{\infty}$ is the norm of the linear functional on $L^{1}$ given by $g$, there exists $f \in L^{1}$ with $\|f\|_{1}^{\infty}=1$ and $|\langle f, g\rangle|>\|g\|_{\infty}-\varepsilon$. By (6), $f=\sum_{n=1}^{\infty} \lambda_{n} p_{a_{n}}$ with $\lambda=\left\{\lambda_{n}\right\} \in l^{1}$ and $\|\lambda\|_{1}<\kappa+\varepsilon$. Therefore

$$
\begin{aligned}
\sup _{z \in D}|g(z)|-\varepsilon & =\|g\|_{\infty}-\varepsilon<\sum_{n=1}^{\infty}\left|\lambda_{n}\right|\left|\left\langle p_{a_{n}}, g\right\rangle\right| \\
& =\sum_{n=1}^{\infty}\left|\lambda_{n}\right|\left|g\left(a_{n}\right)\right| \leqq\|\lambda\|_{1} \sup _{n}\left|g\left(a_{n}\right)\right| \leqq(\kappa+\varepsilon) \sup _{n}\left|g\left(a_{n}\right)\right| .
\end{aligned}
$$


(iii) $\rightarrow$ (iv) $\rightarrow$ (v). $H^{\infty} \subset B H(D)$ and Lemma 1 .

$(v) \rightarrow(v i)$. Brown, Shields and Zeller [2].

(vi) $\rightarrow$ (ii). (See [1]). (ii) $\rightarrow$ (i). Clear.

Remarks. The equality $\|f\|_{1}=\sum_{n=1}^{\infty}\left|\lambda_{n}\right|$ obviously holds if $f=\sum_{n=1}^{\infty} \lambda_{n} p_{a_{n}}$ with $\lambda_{n} \geqq 0$ for all $n$. However there is no sequence $\left\{a_{n}\right\}$ such that this equality holds for all $f \in L^{1}$. For let $f \in L^{1}$ with zero essential infimum on $\partial D$ and $\|f\|_{1}>0$. By taking real parts, we may assume that $f=\sum_{n=1}^{\infty} \lambda_{n} p_{a_{n}}$ with all $\lambda_{n}$ real. If $\lambda_{n} \geqq 0$ for all $n$, then $\lambda_{n} p_{a_{n}} \leqq f$ and so $\lambda_{n}=0$, for all $n$. We may therefore assume that $\lambda_{1}<0$. Then, since $f \geqq 0$ almost everywhere,

$$
\|f\|_{1} \leqq\left\|f+\left|\lambda_{1}\right| p_{a_{1}}\right\|_{1}=\left\|\sum_{n=2}^{\infty} \lambda_{n} p_{a_{n}}\right\|_{1} \leqq \sum_{n=2}^{\infty}\left|\lambda_{n}\right|
$$

Theorem 2 also holds with the disc replaced by the upper half-plane. In fact, the nontrivial step $(\mathrm{v}) \rightarrow(\mathrm{vi})$ is easier to prove in that context and then transfer to $D$ by conformal mapping. See Corollary 5 below.

Notation. Let $U=\{z \in \mathbb{C}: \operatorname{Im} z>0\}$, let $P_{z}(t)$ denote the Poisson kernel for $U$, that is

$$
P_{z}(t)=\frac{1}{\pi} \frac{y}{(x-t)^{2}+y^{2}} \quad(t \in \mathbb{R}, z=x+y i \in U),
$$

and let $|E|$.denote the Lebesgue measure of a measurable set $E$ in $\mathbb{R}$. With $0<\delta<1$, $0<b \leqq \infty, t \in \mathbb{R}$, and $\kappa=\tan (\pi \delta / 2)$, let $\Delta(t, b, \delta)$ denote the triangular domain

$$
\Delta(t, b, \delta)=\{x+y i: \kappa|x-t|<y<b\} .
$$

As usual, the harmonic measure $\chi_{F}(z)$ of a measurable subset $F$ of $\mathbb{R}$ is the harmonic extension to $U$ of the characteristic function $\chi_{F}$, that is

$$
\chi_{F}(z)=\int_{-\infty}^{\infty} \chi_{F}(t) P_{z}(t) d t \quad(z \in U)
$$

Theorem 3. Let $F$ be a Lebesgue measurable subset of $\mathbb{R}$, let $0<\delta<1$, and let $\pi \delta b \geqq|F|$. Then $\chi_{F}(z) \leqq \delta$ for all $z$ in $U \backslash \bigcup_{t \in F} \Delta(t, b, \delta)$.

Proof. As before, we take $\kappa=\tan (\pi \delta / 2)$. If $J=(-\infty, \beta]$ with $\beta$ real, we have for $x>\beta$,

$$
\chi_{J}(z)=\int_{-\infty}^{\beta} P_{z}(t) d t=\frac{1}{\pi} \int_{0}^{y /(x-\beta)} \frac{d u}{1+u^{2}}=\frac{1}{\pi} \arctan \frac{y}{x-\beta}
$$


Thus

$$
0<y \leqq \kappa(x-\beta) \Rightarrow \chi_{J}(z) \leqq \frac{1}{\pi} \arctan \kappa=\frac{\delta}{2}
$$

Similarly, if $J=[\alpha, \infty)$ with $\alpha$ real, then

$$
0<y \leqq \kappa(\alpha-x) \Rightarrow \chi_{J}(z) \leqq \frac{\delta}{2}
$$

Suppose first that $F$ is a closed subset of $\mathbb{R}$, so that $\mathbb{R} \backslash F$ is a countable (perhaps finite or void) union of disjoint open intervals $l_{k}$. Let $z=x+y i \in U \backslash \bigcup_{t \in F} \Delta(t, b, \delta)$ with $0<y<b$. Then $x \in I_{k}$ for some $k$. If $I_{k}=(-\infty, d)$ with $d$ real, we take $J=[d, \infty)$. Since $d \in F, z \notin \Delta(d, b, \delta)$; and, since $y<b$, it follows that $y \leqq \kappa(d-x)$. Since $F \subset J$, we therefore have

$$
\chi_{F}(z) \leqq \chi_{J}(z) \leqq \frac{\delta}{2}
$$

The same inequality holds if $I_{k}=(c, \infty)$. If $I_{k}=(c, d)$ with $-\infty<c<d<\infty$, we take $J=(-\infty, c], J^{\prime}=[d, \infty)$. Since $c \in F$, we have $\chi_{J}(z) \leqq \delta / 2$; and similarly for $\chi_{J^{\prime}}$. Then since $F \subset J \cup J^{\prime}$,

$$
\chi_{F}(z) \leqq \chi_{J}(z)+\chi_{J^{\prime}}(z) \leqq \delta
$$

Finally, if $y \geqq b$, then $P_{z}(t) \leqq 1 / \pi b$ for all real $t$, and so

$$
\chi_{F}(z) \leqq|F| / \pi b \leqq \delta,
$$

and the theorem is proved for closed sets $F$.

Finally, given any Lebesgue measurable subset $F$ of $\mathbb{R}$, there exists an increasing sequence $\left\{F_{n}\right\}$ of closed subsets of $F$ with its union differing from $F$ by a set of measure zero. We have $\chi_{F_{n}}(z) \leqq \delta$ for all $z$ in $U \backslash \bigcup_{t \in F} \Delta(t, b, \delta)$, and the result follows.

Remark. The possibility of a result like Theorem 3 is suggested by the proof in Brown, Shields and Zeller [2] to which we have referred already.

Corollary 4. Let $0<\delta<1$ and let the sequence $\left\{a_{n}\right\}$ of points of $U$ fail to satisfy the following condition: for almost all $t \in \mathbb{R}, \Delta(t, b, \delta) \cap\left\{a_{n}: n \in \mathbb{N}\right\}$ is non-empty for every $b>0$.

Then there exists a positive harmonic function $g$ on $U$ with $\sup _{z \in U} g(z)=1$ but $\sup _{n \in N} g\left(a_{n}\right)<\delta$.

Proof. Let $E=\bigcup_{b>0} A(b)$, with

$$
A(b)=\left\{t \in \mathbb{R}: \Delta(t, b, \delta) \cap\left\{a_{n}: n \in \mathbb{N}\right\}=\emptyset\right\} .
$$


Since $A(b) \supset A\left(b^{\prime}\right)$ when $b<b^{\prime}$, we have $E=\bigcup_{k \in N} A(1 / k)$; and, since each $A(b)$ is closed, it follows that $E$ is measurable. By assumption, we now have $|E|>0$, and so we can choose $b=1 / k$ with $|A(b)|>0$. We take a closed interval $I$ chosen so that, with $F=I \cap A(b)$, we have $0<|F| \leqq \delta \pi b$. Theorem 3 now provides the required function $g=\chi_{F}$.

Corollary 5. Let $\left\{a_{n}\right\}$ be a sequence of points of $U$ such that there exists a constant $\kappa$ with

$$
\sup _{z \in U} g(z) \leqq \kappa \sup _{n \in \mathbb{N}} g\left(a_{n}\right)
$$

for all bounded positive harmonic functions $g$ on $U$. Then almost every point of $\mathbb{R}$ is the non-tangential limit of some subsequence of $\left\{a_{n}\right\}$.

Proof. Immediate consequence of Corollary 4.

Corollary 5 can be transferred to the disc by conformal mapping. It is of interest, because it is not obvious that the inequality (7) for bounded positive harmonic functions $g$ implies the same inequality for all $g \in B H(U)$, though this implication is obvious if $\kappa=1$.

Let $\left\{a_{n}\right\}$ be a sequence of points of $U$, and let $T$ be the bounded linear mapping of $l^{1}$ into $L^{1}=L^{1}(\mathbb{R})$ defined by

$$
T \lambda=\sum_{n=1}^{\infty} \lambda_{n} P_{a_{n}} \quad\left(\lambda=\left\{\lambda_{n}\right\} \in l^{1}\right)
$$

Theorem 2, for $U$ in place of $D$, tells us that $T$ is surjective if and only if $\left\{a_{n}\right\}$ is nontangentially dense for $\mathbb{R}$. It is an immediate consequence that $T$ is never bijective, for if $\left\{a_{n}\right\}$ is non-tangentially dense, then so is $\left\{a_{n+1}\right\}$ and we have

$$
P_{a_{1}}=\sum_{n=2}^{\infty} \lambda_{n} P_{a_{n}}
$$

with $\sum_{n=2}^{\infty}\left|\lambda_{n}\right|<\infty$. In these circumstances, it is natural to ask for what sequences $\left\{a_{n}\right\}$ the mapping $T$ is injective. We do not know the answer to this question, but using an argument due to J. B. Garnett, it is easy to prove the following result.

Theorem 6. $T$ has zero kernel and closed range if and only if $\left\{a_{n}\right\}$ satisfies the geometric condition for $H^{\infty}$ interpolation, that is there exists $\delta>0$ such that

$$
\inf _{k} \prod_{j, j \neq k}\left|a_{k}-a_{j}\right| /\left|a_{k}-\bar{a}_{j}\right| \geqq \delta .
$$

Proof. Since $T \in B L\left(l^{1}, L^{1}\right)$, the usual identification of dual spaces gives $T^{*} \in B L\left(L^{\infty}, l^{\infty}\right)$, and, with $g(z)$ denoting the harmonic extension of $g \in L^{\infty}$ to $U$, we have $T^{*} g=\left\{g\left(a_{n}\right)\right\} \in l^{\infty}$. If $\left\{a_{n}\right\}$ is an interpolation sequence for $H^{\infty}$, then $T^{*} L^{\infty}=l^{\infty}$, and, by 
Banach's closed range theorem [3, p. 488], $T$ has closed range and zero kernel. On the other hand, if $T$ has closed range and zero kernel, then there exists a constant $M$ with

$$
\|\lambda\|_{1} \leqq M\left\|\sum_{n=1}^{\infty} \lambda_{n} P_{a_{n}}\right\|_{1} \quad\left(\lambda=\left\{\lambda_{n}\right\} \in l^{1}\right) .
$$

This is the inequality (4.5) in Garnett $[4$, p. 303] from which it is there deduced that $\left\{a_{n}\right\}$ satisfies the geometric condition for $H^{\infty}$ interpolation.

\section{REFERENCES}

1. F. F. Bonsall, Decompositions of functions as sums of elementary functions, Quart, $J$. Math. Oxford (2), 37 (1986), 129-136.

2. L. Brown, A. Shields and K. Zeller, On absolutely convergent exponential sums, Trans. Amer. Math. Soc. 96 (1960), 162-183.

3. N. Dunford and J. T. Schwartz, Linear Operators Part I (Interscience Publishers, New York, 1958).

4. J. B. Garnett, Bounded Analytic Functions (Academic Press, New York, 1981).

SCHOOL OF Mathematics

UNIVERSITY OF LEEDS

LEEDS LS2 9JT 